\title{
The relation between sleep and weight in a suburban sleep center: observations and speculations on apnea and weight
}

\section{Robert G Hooper}

The Sleep Center, PC, Scottsdale, AZ, USA
This article was published in the following Dove Press journal:

Nature and Science of Sleep

21 November 2016

Number of times this article has been viewed

Study objectives: The relationship between obstructive sleep apnea (OSA) and body weight is not clearly established. In order to describe the relationship of weight and OSA severity seen in a suburban sleep center, an observational review was performed of initial diagnostic polysomnograms (PSGs) ordered on patients with American Academy of Sleep Medicine (AASM) symptomatic indications.

Methodology/principle findings: Initial, full-night diagnostic or initial split-night (diagnostic portion) PSGs performed for any indication on patients $>18$ years old were retrospectively reviewed for a two year period. All studies were performed following AASM guidelines. PSG data were reviewed for the presence and severity of apnea (no OSA - apnea hypopnea index (AHI) <5, mild - AHI 5-14, moderate - AHI 15-29, severe-AHI 30-59, and very severe - AHI $>60$ ). Data were reviewed from 629 PSGs (37\% females and 63\% males) of which 450 met the criteria for apnea. Studies were classified by apnea severity (196 mild, 103 moderate, 91 severe apnea and 60 with very severe apnea) and weight (body mass index (BMI)). Of those with apnea, and BMIs $<25$, severe or very severe apnea occurred in $22 \%(10 / 45)$. Three individuals with BMIs $<20$ had apnea, one severe. Of those with BMIs $\geq 40$, one (1.6\%) did not have apnea and $52 \%(31 / 60)$ had $\mathrm{AHI}>30$.

Conclusion/significance: The profile of this nonrandom series, tested because they were suspected of having a disorder of sleep, provides guidelines for physicians in their approach to symptomatic patients. Individuals with a normal BMI can have apnea, including severe apnea. Severe obesity (BMI >40) is almost always associated with apnea when symptoms are present. Obesity increases the severity of the diagnosed apnea. Excessive weight should be an indication for testing, but normal weight should not exclude individuals with appropriate symptoms. Obesity, while a major contributing factor to severity, is not the etiological cause of OSA in the majority of these patients.

Keywords: apnea, nonobese apnea, apnea severity, etiology of apnea, obesity

\section{Introduction}

The role that body weight plays in the diagnosis, presentation and pathogenesis of obstructive sleep apnea (OSA) remains a subject of discussion. There is little doubt that obesity plays a significant role in the causation and severity of OSA. Population based surveys have reported a strong relationship between weight and the occurrence of OSA. ${ }^{1-5}$ Other reports have noted that weight does not explain OSA in many individuals. ${ }^{3,6-8}$ Although it is known that OSA occurs in the absence of excessive body weight, there is little information for the clinician about those individuals who have sleep-related symptoms but are not obese. Does a symptomatic clinic population reflect
Correspondence: Robert G Hooper The Sleep Center, PC, 10277 North 92nd street, Suite 103, Scottsdale, AZ, USA

Tel + I 48076788 II

Fax + I 4806570737

Email Rhoop@thesleepcenteraz.com 
findings of general population studies? When should body weight play a role in choosing patients for polysomnogram (PSG) testing? Should body weight play a role in declining to test thin, symptomatic individuals? In addition, what role does body weight play as an etiology for OSA?

Polysomnographic studies from a suburban, physician owned, American Academy of Sleep Medicine (AASM) accredited sleep center were reviewed with the goal of establishing a profile of the relationship of weight and apnea in that clinic population. The Ethical Standards Committee of The Sleep Center PC approved this study.

\section{Methods}

Consecutive PSGs performed for an initial sleep evaluation were reviewed. PSG studies for positive airway pressure titration, tests for individuals who had a previous diagnosis of OSA and tests from individuals younger than eighteen years were excluded. All patients studied had been evaluated by a board certified sleep specialist and were studied for a variety of indications that fell under the AASM guidelines. ${ }^{9}$ Both full-night diagnostic and the diagnostic portion of splitnight tests were included. This review of data was carried out retrospectively of a two year period when home sleep apnea tests were not performed. The only clinical information reviewed included body weight (expressed BMI), age, and sex. Consent to utilize test findings for inclusion in studies of PSG results was obtained at the time of testing.

PSGs were performed following the standards of the AASM at the time of the testing. ${ }^{10}$ The parameter measurements reviewed for this report were made by the clinical staff for the clinical use of the physicians and patients. The clinical parameters measured conformed to the recommendation of the AASM at the time of testing. This study reviewed only the PSG results, weight, age and sex of the individuals undergoing testing.

The PSG results were classified by BMI and by the degree of sleep-disordered breathing present (Table 1). The BMI classification used was that of the National Institutes of Health. ${ }^{11}$ The degree of sleep-disordered breathing was determined by the apnea hypopnea index (AHI) reported for the diagnostic portion of the test. The definitions of apneas

Table I Classification of weight and apnea severity

\begin{tabular}{llll}
\hline Weight (BMI) & \multicolumn{3}{c}{ Apnea (AHI) } \\
\hline Normal & $\leq 24$ & Normal & $\leq 4$ \\
Overweight & $25-29$ & Mild & $5-14$ \\
Obese & $30-39$ & Moderate & $15-29$ \\
Severely obese & $\geq 40$ & Severe & $30-59$ \\
& & Very severe & $\geq 60$ \\
\hline
\end{tabular}

Abbreviations: BMI, body mass index; $\mathrm{AHI}$, apnea hypopnea index. and hypopneas used for scoring records were those of the AASM. ${ }^{10}$

\section{Results}

Six hundred twenty-nine PSG results were reviewed. These tests were from 397 (62\%) males and 232 (37\%) females whose BMIs varied from 16.5 to 58.8. There were 450 PSGs with AHIs of equal to or greater than five, which were from 309 (69\%) males and 141 (31\%) females. The AHIs ranged from 5 to 128 . The weight, age and sex distributions of the 450 apnea patients are presented in Figures 1 and 2.

The relationship of weight to the degree of apnea recorded is shown in Table 2. When apnea was present, $43.6 \%$ (196/450) had mild apnea, 22.9\% (103/450) had moderate apnea, $20.2 \%(91 / 450)$ had severe apnea and $13.3 \%$ $(60 / 450)$ had very severe apnea. The weight of the individuals diagnosed with apnea included $45(10 \%)$ with BMIs $<25 ; 141$ (31.4\%) with BMIs 25 to 29; 204 (45.3\%) with BMIs 30 to 39 ; and 60 (13.3\%) with BMIs $\geq 40$.

Nonobese individuals (BMI <30) accounted for $41 \%$ (186/450) of all diagnoses of apnea and 30\% (46/151) of those diagnosed with severe and very severe apnea (AHI $>30)$. Severe or very severe apnea occurred in $22 \%(10 / 45)$ of those individuals with BMIs of $<25$ who had apnea. Three of nine individuals with BMIs $<20$ had apnea, one severe.

Severely obese individuals (BMI of $\geq 40$ ) had apnea $98 \%$ $(60 / 61)$ of the time. The apnea of the severely obese was rated mild $30 \%(18 / 60)$, moderate $18.3 \%$ (11/60), severe $26.7 \%$ $(16 / 60)$, and very severe $25 \%(15 / 60)$.

Severe or very severe apnea occurs in all weight groups (Figure 3). In the PSGs with apnea, it was diagnosed in $22.2 \%$ $(10 / 45)$ of those with BMIs <25; in 25.5\% (36/141) of those with BMIs of 25 to 29 ; in $36.3 \%$ (74/204) of those with BMIs 30 to 39 ; and in $51.6 \%(31 / 60)$ of those with BMIs $\geq 40$.

\section{Discussion}

Excessive body weight has long been associated with the diagnosis of OSA. The recognition of its importance has led to concern about the frequency of OSA and severity of apnea in individuals with weight problems. While it is recognized that apnea can occur in individuals who are not obese, the relative number of these individuals seen is not generally recognized. ${ }^{1,3,6}$ Furthermore, the severity of apnea observed at different weight levels is not defined. ${ }^{3,12}$ This observational experience sheds some light on these issues.

This report confirms that apnea occurs at all weight levels (Table 2). In the absence of obesity, overweight and normal weight individuals can have apnea and it can be severe. 
Sex, weight, and apnea severity

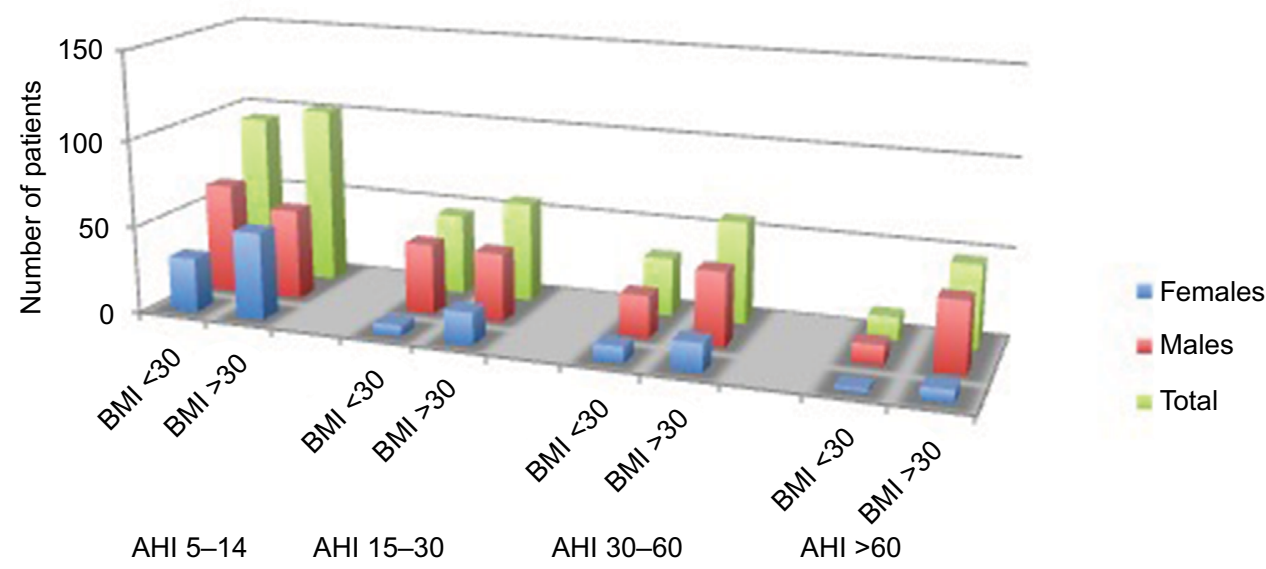

Figure I The distribution of patients by sex, weight and apnea severity. Abbreviations: $\mathrm{BMI}$, body mass index; $\mathrm{AHI}$, apnea hypopnea index.

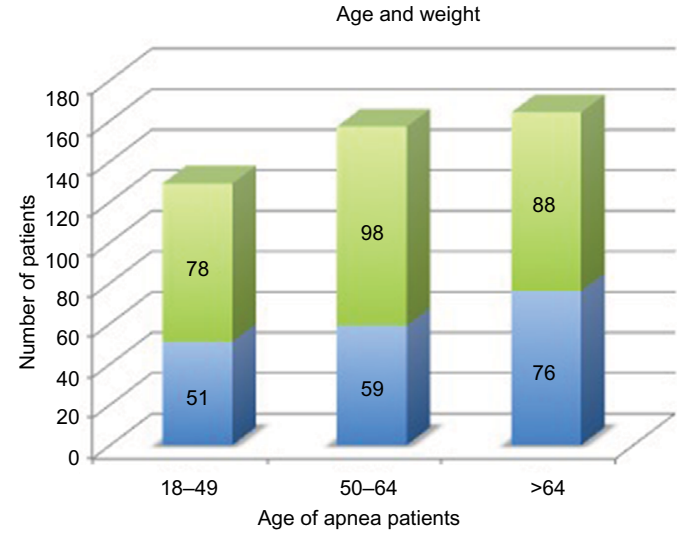

Figure 2 The distribution of patients by age and weight.

These statements are neither surprising nor do they represent new findings. Population studies have demonstrated that those with apnea are obese a majority of the time..$^{1-6,12}$ Our symptomatic patients were similar with $59 \%$ obese. These observations do suggest that OSA may be more of a problem in the nonobese than may be generally appreciated. Severe apnea was observed in $22 \%$ of those with a BMI of $<25$ in our referred patients suspected of having a sleep disorder. In the proper situation, apnea should be suspected in the nonobese individual.

Severe obesity (BMI $\geq 40$ ) has a strong relationship with apnea. In studies of patients referred for bariatric surgery, when all patients with BMIs of greater than 40 are studied routinely without regard to the presence of symptoms, apnea is present in greater than $70 \% .^{3,13,14}$ The referral base for this study population did not include a bariatric program. Our symptomatic patients with severe obesity (BMIs $\geq 40$ ) had some degree of apnea $98 \%$ of the time, but were severe
Table 2 Relation of weight to $\mathrm{AHI}$

\begin{tabular}{|c|c|c|c|c|c|c|}
\hline \multicolumn{7}{|c|}{ BMI and apnea severity } \\
\hline \multirow[t]{2}{*}{ BMI } & \multicolumn{3}{|c|}{$\mathrm{AHI}(\mathrm{n})$} & & \multicolumn{2}{|c|}{ Total } \\
\hline & $5-14$ & $15-29$ & $30-59$ & $\geq 60$ & & \\
\hline$\leq 24$ & 24 & 11 & 7 & 3 & 45 & $10 \%$ \\
\hline $25-29$ & 70 & 35 & 26 & 10 & $|4|$ & $31 \%$ \\
\hline $30-39$ & 84 & 46 & 42 & 32 & 204 & $45 \%$ \\
\hline$\geq 40$ & 18 & $\mathrm{II}$ & 16 & 15 & 60 & $13 \%$ \\
\hline Totals & 196 & 103 & 91 & 60 & 450 & $100 \%$ \\
\hline \multicolumn{7}{|c|}{ Nonobese/obese and apnea severity } \\
\hline \multirow[t]{2}{*}{ BMI } & \multicolumn{3}{|c|}{ AHI (n) } & & \multicolumn{2}{|c|}{ Total } \\
\hline & $5-14$ & $15-29$ & $30-59$ & $\geq 60$ & & \\
\hline$\leq 29$ & 94 & 46 & 33 & 13 & 186 & $41 \%$ \\
\hline$\geq 30$ & 102 & 57 & 58 & 47 & 264 & $59 \%$ \\
\hline Totals & 196 & 103 & 91 & 60 & 450 & $100 \%$ \\
\hline
\end{tabular}

Abbreviation: BMI, body mass index; AHI, apnea-hypopnea index.

only $51 \%$ of the time. Previous reports of the distribution of apnea severity in massively obese individuals could not be found by the author.

The value of this work is limited by several factors. The data collected are observational in nature. Patient clinical data other than sex, age and weight were not reviewed. However, at the time of testing, a board certified sleep physician reviewed all patient data and the patients were felt to have had appropriate indications and symptoms for PSG testing. The patient population consisted of individuals referred from private practices in a suburban community. The study data were from clinical patient care testing that by necessity involved multiple technicians and scoring staff. These data report the AHI that represents the overall AHI results for the diagnostic portion of the studies. The effects of positional and sleep stage changes on the AHI were not analyzed for this report. The findings may be explained by the observational 


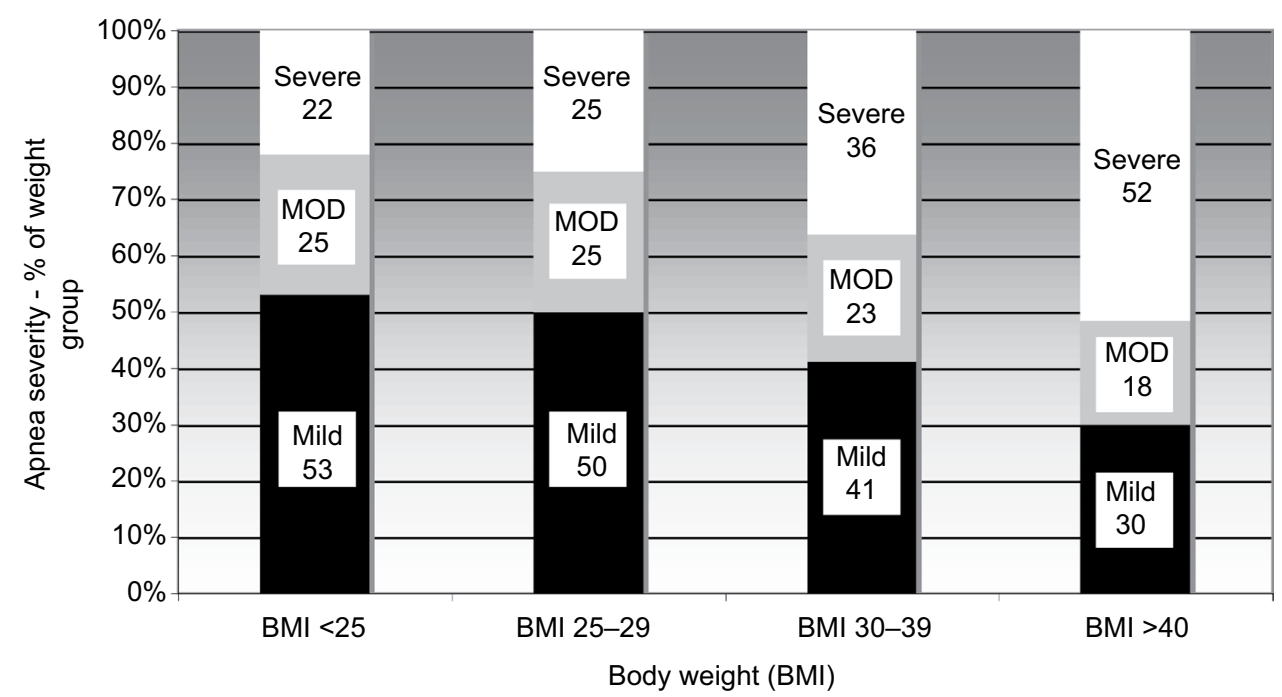

Figure 3 Columns represent the distribution of apnea severity for each weight group. Black represents the percentage of mild (AHI 5-I4), gray represents moderate (MOD) (AHI I5-29), and white represents severe and very severe (AHI $\geq 30$ ) apnea. The number of patients the distributions reflect are 45 (BMI $<25$ ), I4I (BMI $25-29), 204$ (BMI 30-39), and 60 (BMI $\geq 40$ ).

Abbreviations: BMI, body mass index; AHI, apnea hypopnea index.

approach used in this report. A referral bias based upon the type of patients evaluated could explain these findings; individuals referred may represent thinner than average or heavier than average.

Obstructive apnea is the result of collapse of the upper airway in the oropharynx and/or nasal pharynx during sleep. The two determining factors of that collapse are the underlying upper airway anatomy (size, shape, and geometry) and the airway tone (tissue and muscular). ${ }^{15}$ Multiple mechanisms have been postulated as sources of the airway collapse including anatomic (bony and soft tissue), respiratory control (changes in $\mathrm{PaCO} 2$ sensitivity and ventilator load response), neuromuscular (changes in pharyngeal airway resistances and muscle tone during sleep), vascular volume with rostral fluid shifts and hormonal. ${ }^{15-19}$ How each of these mechanisms influences the anatomy and airway tone independently and in conjunction with one another is not definitively understood.

Obesity is a known major cause and a complicating or aggravating feature of OSA. Obstructive apnea can resolve with weight loss. OSA improves with weight loss and worsens with weight gain. ${ }^{4}$ The evidence for these obesity effects on OSA has been clearly and repeatedly documented. The mechanism(s) by which obesity affects anatomy and tone may be singular or multiple. Anatomic narrowing is of major importance, but is probably not the only mechanism. Are there other causes for the apnea or does the obesity lead to unrecognized permanent changes in anatomy or tone in those who have persistent apnea after weight loss?
This experience, although observational in symptomatic patients referred for testing, provides some insight into the contribution of obesity as the causation of apnea for these and similar individuals. Young et al in their review of the subject speculated that $58 \%$ of all moderate and severe apnea (AHI $>15$ ) can be "attributed" to obesity. ${ }^{12}$ Our clinical population of symptomatic patients with similar AHIs demonstrated a strikingly similar rate of obesity (59\%). While we observed that rate of obesity, it does not mean that obesity was the cause of the apnea or that apnea could be solely attributed to obesity in $59 \%$ of those patients. What relative portion of obstructive apnea can be attributed solely to obesity and what portion is obesity a complicating feature that may worsen underlying obstructive apnea?

Numerous previous studies and reviews have documented the success of weight loss on correcting apnea. There is considerable variation in the study designs, number of patients, and definitions of improvement or resolution following weight loss. ${ }^{3,4,20-24}$ From these studies, it is clear that weight loss usually improves apnea and may result in its resolution. The frequency of OSA resolution (post-weight loss AHI $<5)$ cannot be determined from published work, but appears to occur in less than one-third of patients and two studies suggest it is very low. ${ }^{21,24}$

Using these estimates to reflect on the cause of apnea in these cases we reviewed for this paper, an interesting and provocative observation is present. If $30 \%$ is assumed to be an accurate estimate of OSA resolution after weight loss, then following weight loss, a large percentage of ex-obese patients will still have apnea. If all of our $58.6 \%$ obese 
patients lost weight, then two-thirds of them will still have apnea. Only $17.8 \%$ of the total population will have the apnea resolved with weight loss. The data suggest that at least $80 \%$ of the patients who were represented by these studies have OSA for reasons other than obesity. In this clinic population, obesity is likely a contributing factor, but not the basic cause of apnea in the majority of patients. This speculation has to be tempered by the observational nature of the series and lack of clinical information on the suspected reason for apnea in these patients. In addition, some overweight but not obese patients may experience resolution of apnea with weight loss. But the observations suggest that the majority of OSA patients in this type of patient population have sleep-disordered breathing as the result of factors other than obesity. The author agrees with those who conclude that weight loss is extremely important for many reasons, but that it should be considered an adjunct therapy for apnea. ${ }^{7}$ Primary therapy for apnea should be directed at resolving the episodic nocturnal airway obstructions.

Speculating further, these results suggest the effect that weight loss will have on apnea severity in a population of obese individuals with apnea. Weight loss clearly reduces AHI in some portion, probably a sizable portion, of the obese patient population. After weight loss, some of the formerly obese individuals will not have apnea while others will continue to have some degree of apnea. The postweight loss population of those who continue to have apnea will likely show the distribution of apnea severity seen in our patient groups with BMIs of less than 25 or between 25 and 29 (Figure 3). After weight loss, some patients will continue to have severe apnea, but, milder degrees of apnea will predominate.

This experience demonstrates that apnea occurs in all weight classifications. Symptomatic, severely obese individuals almost always have apnea. Severe obesity (BMI $\geq 40$ ) should be considered an indication for testing. Obesity increases the severity of the apnea diagnosed. Thin individuals can have apnea and can have severe apnea. Symptomatic thin individuals should not be excluded from testing because of body weight.

Reasons other than obesity play a major role in the causation of apnea. ${ }^{15}$ Apnea and obesity are both major health problems. Physicians should view obstructive apnea and obesity as independent clinical problems. While treatment of one may lead to improvement or resolution of the other, patients and clinicians should be aware that obstructive apnea is an issue that most often occurs for other less well understood reasons. Obstructive apnea should be in the differential diagnoses of symptomatic individuals regardless of their weight.

\section{Disclosure}

The author is the owner and medical director of The Sleep Center, PC located in Scottsdale, Arizona, USA. The author is solely responsible for this work and has no financial or other conflicts of interests to report.

\section{References}

1. Kripke DF, Ancoli-Israel S, Klauber MR, Wingard DL, Mason WJ, Mullaney DJ. Prevalence of sleep-disordered breathing in ages 40-64 years: a population based survey. Sleep. 1997;20(1):65-76.

2. Young T, Palta M, Dempsey J, Skatrud J, Weber S, Badr S. The occurrence of sleep-disordered breathing among middle-aged adults. N Engl J Med. 1993;328(17):1230-1235.

3. Tuomilehto H, Seppa J, Uusitupa M. Obesity and obstructive sleep apnea--clinical significance of weight loss. Sleep Med Rev. 2013;17(5): 321-329.

4. Peppard PE, Young T, Palta M, Dempsey J, Skatrud J. Longitudinal study of moderate weight change and sleep-disordered breathing. JAMA. 2000;284(23):3015-3021.

5. Wolk R, Shamsuzzaman AS, Somers VK. Obesity, sleep apnea, and hypertension. Hypertension. 2003;42(6):1067-1074.

6. Young T, Shahar E, Nieto FJ, et al. Predictors of sleep-disordered breathing in community-dwelling adults: the Sleep Heart Health Study. Arch Intern Med. 2002;162(8):893-900.

7. Barvaux VA, Aubert G, Rodenstein DO. Weight loss as a treatment for obstructive sleep apnoea. Sleep Med Rev. 2000;4(5):435-452.

8. Tishier PV, Larkin EK, Schluchter MD, Redline S. Incidence of sleepdisordered breathing in an urban adult population: the relative importance of risk factors in the development of sleep-disordered breathing. JAMA. 2003;289(17):2230-2237.

9. Kushida CA, Littner MR, Morgenthaler T, et al. Practice parameters for the indications for polysomnography and related procedures: an update for 2005. Sleep. 2005;28(4):499-521.

10. Iber C, Ancoli-Israel S, Chesson AL Jr, Quan SF. The AASM Manual for the Scoring of Sleep and Associated Events: Rules, Terminology and Technical Specifications. American Academy of Sleep Medicine, 2007.

11. National Institutes of Health. Clinical Guidelines for the Identification, Evaluation, and Treatment of Overweight and Obesity in Adults. Bethesda, MD: National Institutes of Health; 1998. Available from: http://www.nhlbi.nih.gov/guidelines/obesity/ob_gdlns.pdf. Accessed September 24, 2016.

12. Young T, Peppard PE, Taheri S. Excess weight and sleep-disordered breathing. J Appl Physiol (1985). 2005;99(4):1592-1599.

13. Lopez PP, Stefan B, Schulman CR, Byers PM. Prevalence of sleep apnea in morbidly obese patients who presented for weight loss surgery evaluation: more evidence for routine screening for obstructive sleep apnea before weight loss surgery. Am Surg. 2008;74(9):834-838.

14. O'Keeffe T, Patterson EJ. Evidence supporting routine polysomnography before bariatric surgery. Obes Surg. 2004;14(1):23-26.

15. Dempsey JA, Veasey SC, Morgan BJ, O'Donnell CP. Pathophysiology of sleep apnea. Physiol Rev. 2010;90(1):47-112.

16. Schwab RJ, Pasirstein M, Pierson R, et al. Identification of upper airway anatomical risk factors for obstructive sleep apnea with volumetric magnetic resonance imaging. Am J Respir Crit Care Med. 2003;168(5): 522-530.

17. Dempsey JA, Skatrud JB, Jacques AJ, et al. Anatomic determinants of sleep-disordered breathing across the spectrum of clinical and nonclinical male subjects. Chest. 2002;122(3):840-851.

18. Rowley JA, Zahn BR, Babcock MA, Badr MS. The effect of rapid eye movement (REM) sleep on upper airway mechanics in normal human subjects. J Physiol. 1998;510(Pt 3):963-976. 
19. White LH, Lyons OD, Yadollahi, Ryan CM, Bradley TD. Effect of below-the-knee compression stockings of severity on obstructive sleep apnea. Sleep Med. 2015;16(2)258-264.

20. Valencia-Flores M, Orea A, Herrera M, et al. Effect of bariatric surgery on obstructive sleep apnea and hypopnea syndrome, electrocardiogram, and pulmonary arterial pressure. Obes Surg. 2004;14(6):755-762.

21. Lettieri CJ, Eliasson AH, Greenburg DL. Persistence of obstructive sleep apnea after surgical weight loss. J Clin Sleep Med. 2008;4(4): 333-338.
22. Haines KL, Nelson LG, Gonzalez R, et al. Objective evidence that bariatric surgery improves obesity-related obstructive sleep apnea. Surgery. 2007;141(3):354-358.

23. Fritscher LG, Canani S, Mottin CC, et al. Bariatric surgery in the treatment of obstructive sleep apnea in morbidly obese patients. Respiration. 2007;74(6):647-652.

24. Barnes M, Goldsworthy UR, Cary BA, Hill CJ. A diet and exercise program to improve clinical outcomes in patients with obstructive sleep apnea--a feasibility study. J Clin Sleep Med. 2009;5(5):409-415.

\section{Publish your work in this journal}

Nature and Science of Sleep is an international, peer-reviewed, open access journal covering all aspects of sleep science and sleep medicine, including the neurophysiology and functions of sleep, the genetics of sleep, sleep and society, biological rhythms, dreaming, sleep disorders and therapy, and strategies to optimize healthy sleep. The manuscript
Dovepress

management system is completely online and includes a very quick and fair peer-review system, which is all easy to use. Visit http://www. dovepress.com/testimonials.php to read real quotes from published authors. 